\title{
Massive Thrombus in a Patient With Peripartum Fulminant Myocarditis
}

\author{
Koichiro Imai, MD, Nozomi Watanabe, MD, Nozomi Wada, MD, \\ Takahiro Kawamoto, MD, Yoji Neishi, MD, Hiroyuki Okura, MD and \\ Kiyoshi Yoshida, MD \\ Department of Cardiology Kawasaki Medical School, Kurashiki, Japan
}

\begin{abstract}
Summary
A 28 year-old Japanese woman presented with symptoms of a common cold and visited her primary care physician 60 days after giving birth to her first baby. A few days later, she was brought to our institution in the state of cardiac shock, with fulminant myocarditis. Echocardiography showed severely reduced cardiac systolic function. The electrocardiogram revealed complete atrioventricular block with ST-T wave changes. She underwent mechanical support with percutaneous cardiopulmonary support, intra-aortic balloon pump and left ventricular assist system. However, a massive thrombus was found within all the cardiac chambers (left ventricle, left atrium, right ventricle and right atrium) and the patient died on day 9 of hospitalization.

(J Echocardiogr 2008; 3: 87-89)
\end{abstract}

Key words: Acute myocarditis, peripartum, thrombus

\section{Introduction}

Fulminant myocarditis is a mortal disease which is presented by rapidly intractable cardiac shock. Mechanical support [intra-aortic balloon pump (IABP), percutaneous cardiopulmonary support (PCPS) and left ventricular assist system (LVAS)] is a potentially effective therapeutic option to save those patients' lives and to help them recover and return to their normal lives after being rescued from the fatal cardiopulmonary crisis $[1,2]$. In this report we present our case of peripartum fulminant myocarditis.

\section{Case}

A 28-year-old woman, 8 weeks after giving birth to her first baby, visited her primary care physician because of general fatigue and fever. She was diagnosed with a common cold and was prescribed oral aspirin and antibiotics. Over the next several days, she became dyspneic and was admitted to our hospital.

Received September 19, 2008

Address for correspondence: Nozomi Watanabe, MD

Department of Cardiology Kawasaki Medical School,

577 Matsushima, Kurashiki, 701-0192, Japan.

Telephone: +81-86-462-1111

Fax: +81-86-464-4060

E-mail: non@med.kawasaki-m.ac.jp

(C) 2008 Japanese Society of Echocardiography
She had no previous history of cardiac diseases. On admission, her consciousness was clear; blood pressure (BP) was $78 / 48 \mathrm{mmHg}$; body temperature was $38.3^{\circ} \mathrm{C}$; and heart rate was 62 beats $/ \mathrm{min}$. Cardiac auscultation revealed a third heart sound. Chest auscultation revealed mild coarse crackles over both lower lung fields. Laboratory data showed an increased Creactive protein $(\mathrm{CRP}=2.08 \mathrm{mg} / \mathrm{dl})$ and creatinine kinase concentration (1655 IU/L, CKMB=144IU/L). Echocardiography showed severe systolic dysfunction with a diffusely thickened ventricular wall. Left ventricular ejection fraction (LVEF) was 20\%. Thickness of the intraventricular septum was $22 \mathrm{~mm}$ and LV posterior wall was $23 \mathrm{~mm}$ (Figure. 1). Electrocardiogram (ECG) revealed complete atrioventricular block with ST-segment elevation in leads $\mathrm{V}_{1-4}$ and ST-segment depression in leads $\mathrm{V}_{5-6}$ (Figure. 2-A). Thirty minutes after arriving at the emergency room, the patient's systolic BP rapidly decreased to $48 \mathrm{mmHg}$. Coronary angiography did not reveal any coronary artery stenosis. The patient was diagnosed with acute fulminant myocarditis. Mechanical support using IABP and PCPS was immediately started under mechanical ventilation with intravenous catecholamine and high-dose gamma-globulin. On day 5 of hospitalization, ECG revealed cardiac arrest (Figure. 2-B). Transthoracic and transesophageal echocardiography showed deteri- 


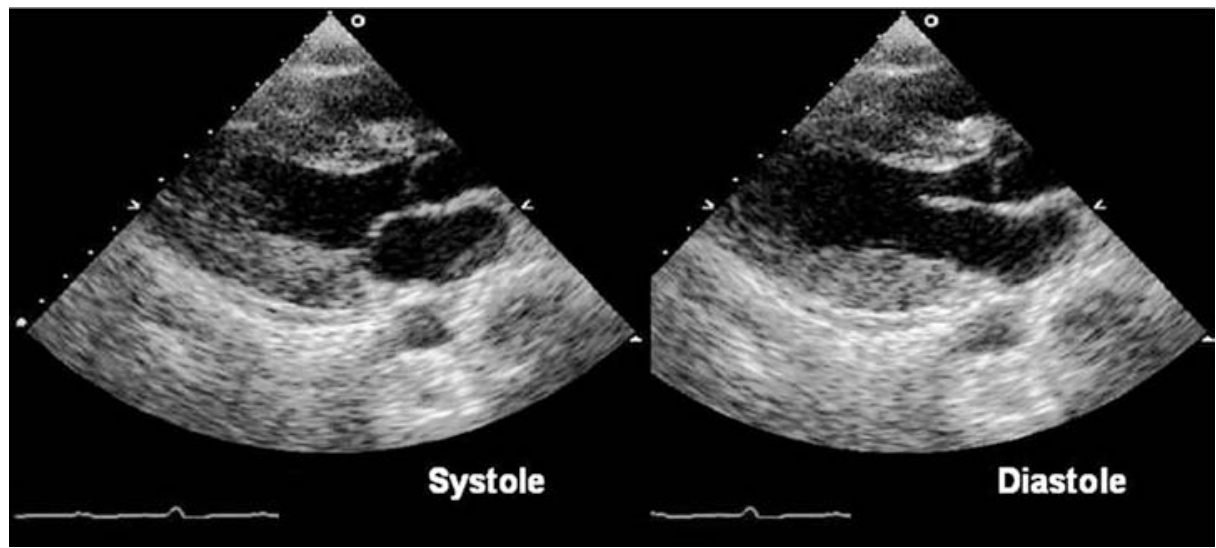

Fig. 1 Echocardiograms on admission showing edematous thickened left ventricular wall. (Long-axis view, Left: Systole, Right: Diastole)
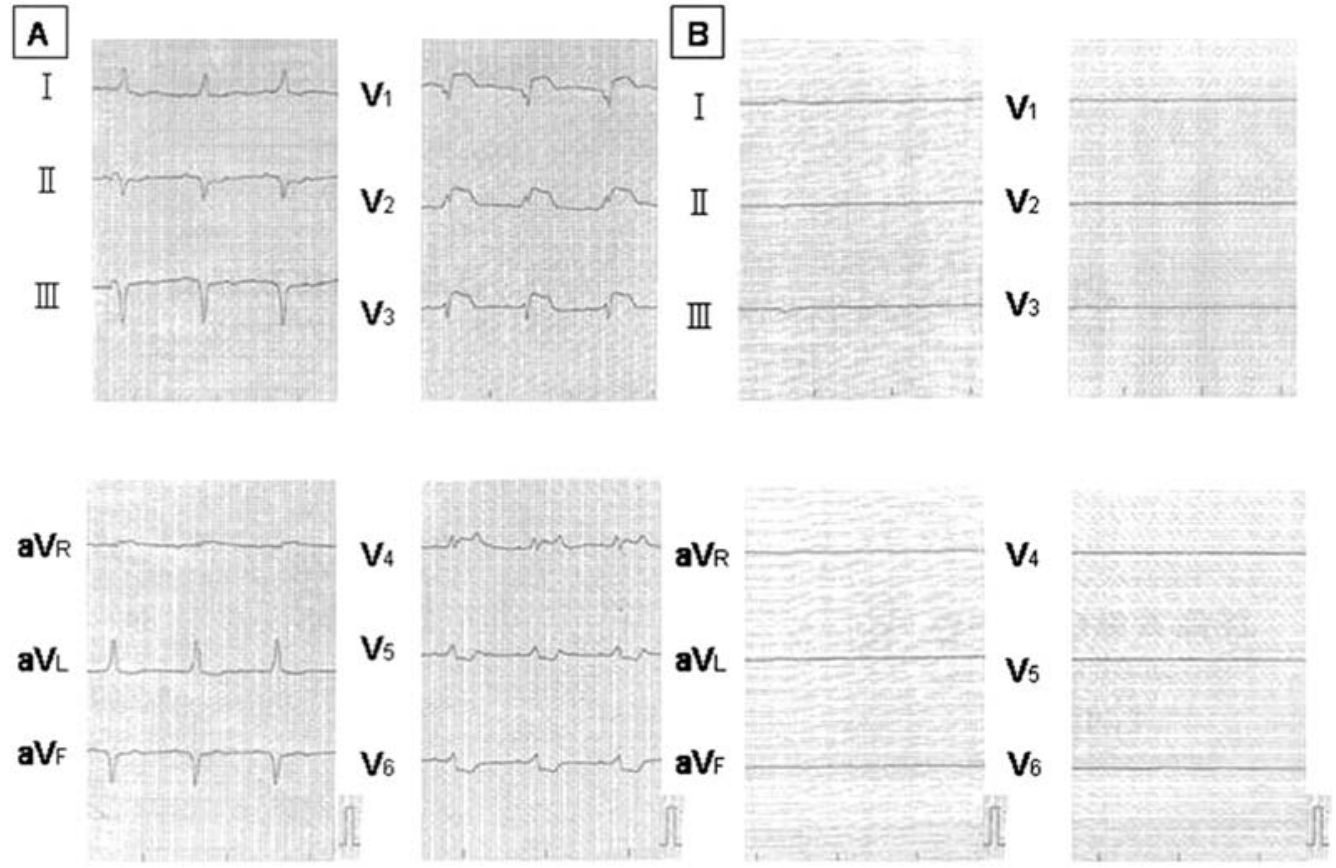

Fig. 2-A Electrocardiogram recorded on admission showing complete atrioventricular block with ST-segment elevation in leads $V_{1-4}$ and ST-segment depression in leads $V_{5-6}$.

Fig. 2-B Electrocardiogram recorded on day 5 of hospitalization showing cardiac asystole.

orated LV wall motion and thrombotic tendency inside all the cardiac chambers [left ventricle (LV), left atrium (LA), right ventricle (RV) and right atrium (RA) ] (Figure 3A 3D). Her condition did not improve, and we implanted an LVAS on day 8 of hospitalization. However, the patient died of multiple organ failure the following day.

\section{Discussion}

Acute myocarditis exhibits various clinical presentations ranging from asymptomatic to cardiac shock. In this case, although we immediately started mechanical support, we were unable to save the patient's life.

Previous reports have suggested that peripartum cardiomyopathy is rare, occurring approximately once in every 2500 deliveries and comprises less than $1 \%$ of pregnancy-associated cardiovascular abnormalities [1]. The etiology remains unclear, but Midei et al reported that myocarditis was present in $78 \%$ of peripartum cardiomyopathies [3].

In the present case study, the cardiac chambers were occupied by a massive thrombus on day 5 of hospitalization despite appropriate use of PCPS and anticoagulation. There are some reports about the frequen- 


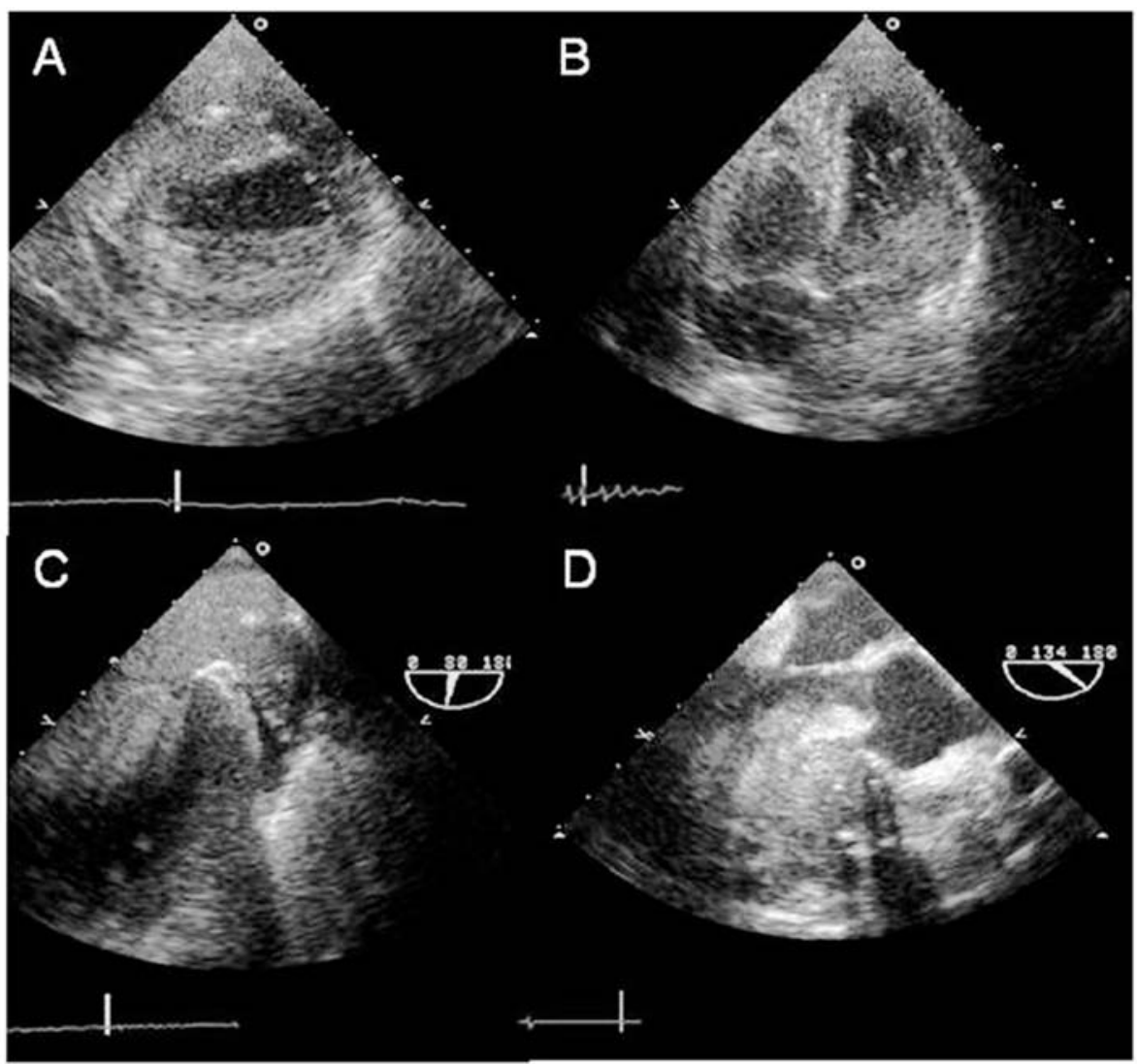

Fig. 3-A Transthoracic echocardiography on day 5 of hospitalization after introduction of percutaneous cardiopulmonary support showing that a massive thrombus had developed within the left ventricle (LV) (Short-axis view).

Fig. 3-B Transthoracic echocardiography on day 5 of hospitalization after introduction of percutaneous cardiopulmonary support showing that the massive thrombus had developed and occupied all the cardiac chambers [LV, left atrium (LA), right ventricle (RV) and right atrium (RA)] (Four-chamber view).

Fig. 3-C Transesophageal echocardiography on day 5 of hospitalization showing a thrombus in the left atrium and left atrial appendage.

Fig. 3-D Transesophageal echocardiography on day 5 of hospitalization showing a thrombus in the LV and left ventricular outflow tract.

cy of thrombus formation inside the cardiac chambers in acute myocarditis which is considered to be secondary to LV dysfunction $[4,5]$. Miyamoto et al reported a massive thrombus formation with heparin-induced thrombocytopenia (HIT) in patients with fulminant myocarditis [6]. Our case did not show any evidence of HIT throughout the hospitalization, and we think that the massive thrombus formation in our patient occurred as a result of rapid LV dysfunction.

\section{References}

1. McCarthy RE, 3rd, Boehmer JP, Hruban RH, et al. Longterm outcome of fulminant myocarditis as compared with acute (nonfulminant) myocarditis. N Engl J Med 2000; 342(10): 690-5.

2. Kato S, Morimoto S, Hiramitsu S, Nomura M, Ito T,
Hishida H. Use of percutaneous cardiopulmonary support of patients with fulminant myocarditis and cardiogenic shock for improving prognosis. Am J Cardiol 1999; 83(4): 623-5, A10.

3. Midei MG, DeMent SH, Feldman AM, Hutchins GM, Baughman KL. Peripartum myocarditis and cardiomyopathy. Circulation 1990; 81(3): 922-8.

4. Pinamonti B, Alberti E, Cigalotto A, Dreas L, Salvi A, Silverstri F. Echocardiographic findings in myocarditis. Am J Cardiol. 1996; 62: 285-91.

5. Ikaheimo MJ, Takkunen JT. Echocardiography in acute infectious myocarditis. Chest. 1986; 89: 100-2.

6. Miyamoto K, Yasuda S, Noguchi T, et al. Fulminant myocarditis causing severe left heart failure and massive thrombus formation following cardiac tamponade: a case report. J Cardiol 2005; 46(1): 25-31. 